\title{
On the strength of mutual stationarity
}

\author{
Peter Koepke \\ Mathematisches Institut der Universität Bonn, Beringstraße 6, D-53115 Bonn, Germany \\ Philip Welch \\ School of Mathematics, University of Bristol, Bristol BS8 1TW, England
}

April 26, 2005

\begin{abstract}
For $\left(\kappa_{n}\right)_{n<\omega}$ a strictly increasing sequence of regular cardinals $\geqslant \aleph_{2}$, Foreman and Magidor showed: if every sequence $\left(S_{n}\right)_{n<\omega}$ of sets $S_{n}$, which are stationary in $\kappa_{n}$ with $\forall \xi \in S_{n} \operatorname{cof}(\xi)=\omega_{1}$, is mutually stationary then $V \neq L$. We show that the existence of a sequence $\left(\kappa_{n}\right)_{n<\omega}$ with this property is equiconsistent with the existence of a measurable cardinal. In case $\left(\kappa_{n}\right)_{n<\omega}=\left(\aleph_{n+3}\right)_{n<\omega}$ the property implies the existence of inner models with many measurable cardinals .
\end{abstract}

\section{Introduction}

The concept of mutual stationarity was introduced by M. ForEman and M. MAGIDOR [4] in order to transfer some combinatorial aspects of stationary subsets of regular cardinals to singular cardinals. Together with J. Cummings they further investigated the status of such sequences in [3].

Definition 1 Let $\left(\kappa_{n}\right)_{n<\omega}$ be a strictly increasing sequence of regular cardinals $\geqslant \aleph_{2}$ with $\kappa_{\omega}=\sup _{n<\omega} \kappa_{n}$. A sequence $\left(S_{n}\right)_{n<\omega}$ is called mutually stationary in $\left(\kappa_{n}\right)_{n<\omega}$ if every first-order structure $\mathfrak{A}$ of countable type with $\kappa_{\omega} \subseteq \mathfrak{A}$ has an elementary substructure $\mathfrak{B} \prec \mathfrak{A}$ such that $\forall n<\omega \sup |\mathfrak{B}| \cap \kappa_{n} \in S_{n}$.

Note that if $\left(S_{n}\right)_{n<\omega}$ is mutually stationary in $\left(\kappa_{n}\right)_{n<\omega}$ then each $S_{n} \cap \kappa_{n}$ is stationary in $\kappa_{n}$. In the following we shall denote the class $\{\xi \in \operatorname{Ord} \mid \operatorname{cf}(\xi)=\lambda\}$ by $\operatorname{Cof}_{\lambda}$. For $X \subseteq$ Ord a set, we write ot $(X)$ for its order type.

Definition 2 Let $\left(\kappa_{n}\right)_{n<\omega}$ be a strictly increasing sequence of regular cardinals and $\lambda<\kappa_{0}, \lambda$ regular. The mutual stationarity property $\operatorname{MS}\left(\left(\kappa_{n}\right)_{n<\omega}, \lambda\right)$ is the statement: if $\left(S_{n}\right)_{n<\omega}$ is a sequence of sets $S_{n} \subseteq \operatorname{Cof}_{\lambda}$ which are stationary in $\kappa_{n}$ then $\left(S_{n}\right)_{n<\omega}$ is mutually stationary in $\left(\kappa_{n}\right)_{n<\omega}$. 
M. Foreman and M. MAGidor [4] proved the following two theorems:

Theorem. For $\left(\kappa_{n}\right)_{n<\omega}$ a strictly increasing sequence of uncountable regular cardinals, $\operatorname{MS}\left(\left(\kappa_{n}\right)_{n<\omega}, \omega\right)$ holds.

Theorem. $\operatorname{MS}\left(\left(\kappa_{n}\right)_{n<\omega}, \omega_{1}\right)$ implies $V \neq L$.

In fact, they proved much more in the latter theorem: assuming $V=L$ they exhibited a double-indexed sequence $S_{n}^{h} \subseteq \omega_{n+2}(n<\omega, 1 \leq h<\omega)$, where each $S_{n}^{h}=\operatorname{Defcol}(h) \cap \operatorname{Cof}_{\omega_{1}}$. For $\alpha$ not a cardinal, let $\beta(\alpha)+1$ be the least level of the $L$-hierarchy where $\alpha$ is singular, and let $h(\alpha)$ be the least level of definability, so that there is a $\Sigma_{h}\left(L_{\beta(\alpha)}\right)$ definable function witnessing the singularity of $\alpha$. Then $\operatorname{Defcol}(h)=\{\alpha \in \operatorname{Ord} \mid h(\alpha)=h\}$. Their result then is: For any function $f: \omega \longrightarrow \omega,\left\langle S_{n}^{f(n)}\right\rangle$ is mutually stationary if and only if $f$ is eventually constant.

We strengthen this to:

Theorem 1 The theories ZFC $+\exists\left(\kappa_{n}\right)_{n<\omega} \mathrm{MS}\left(\left(\kappa_{n}\right)_{n<\omega}, \omega_{1}\right)$ and ZFC $+\exists \kappa(\kappa$ measurable) are equiconsistent.

The implication from right to left was proved by J. Cummings, Foreman, and Magidor [3] via Prikry forcing. Again they proved more than this: they showed that a tail of the Prikry generic sequence satisfies $\operatorname{MS}\left(\left(\kappa_{n}\right)_{n<\omega}, \lambda\right)$ for any $\lambda<\kappa_{0}$ (or indeed the mutual stationarity of any sequence of stationary sets $S_{n} \subseteq \kappa_{n}$ irrespective of the cofinalities of the ordinals in the $S_{n}$.) This is essentially obtained by utilising the fact that a tail of the Prikry generic sequence remains coherently Ramsey in the generic extension. The converse which we prove here uses the core model $K$ of A. J. DodD and R. B. Jensen (see [2]). We deduce the existence of $O^{\sharp}$ from $\operatorname{MS}\left(\left(\kappa_{n}\right)_{n<\omega}, \omega_{1}\right)$ in detail. The proof involves the global square principle $\square$ in $L$ and techniques from the JENSEN Covering theorem for $L$ (see [1]). Fine structural details will be presented in the hyperfine structure theory of S. D. FRIEDMAN and the first author [5]. Although the hyperfine structure for the Dodd-Jensen Core Model is not yet published we shall nevertheless indicate how to transfer the arguments from $L$ to the Dodd-Jensen $K$ for the proof of the full theorem.

In case $\left(\kappa_{n}\right)_{n<\omega}$ consists of "small" cardinals we can obtain higher consistency strengths:

Theorem 2 If $\operatorname{MS}\left(\left(\aleph_{n+3}\right)_{n<\omega}, \omega_{1}\right)$ holds then there is an inner model with infinitely many measurable cardinals $\kappa$ of Mitchell order $o(\kappa)=\omega_{1}$.

Better results than the above are obtainable, but we leave the precise statement (and a proof of Theorem 2) to a later paper. For these results, the hyperfine structure theory has not been developed, and so there recourse is made to more standard fine structure.

\section{Order types of Square Sequences}

Definition 3 Let $\operatorname{Sing}=\{\beta \in \operatorname{Ord} \mid \lim (\beta) \wedge \operatorname{cf}(\beta)<\beta\}$ be the class of singular limit ordinals. Global square $(\square)$ is the assertion: there is a system $\left(C_{\beta}\right)_{\beta \in \operatorname{Sing}}$ 
satisfying:

(a) $C_{\beta}$ is a closed cofinal subset of $\beta$;

(b) ot $\left(C_{\beta}\right)<\beta$;

(c) if $\bar{\beta}$ is a limit point of $C_{\beta}$ then $\bar{\beta} \in \operatorname{Sing}$ and $C_{\bar{\beta}}=C_{\beta} \cap \bar{\beta}$.

Jensen [6] introduced the principle $\square$ and proved it in $L$. The second author [8] proved $\square$ in the Dodd-Jensen core model $K$. From the order types of the square sequences $C_{\xi}$ we shall define stationary sets $S_{n}$ to which we shall apply the MS-principle.

Theorem 3 Let $\kappa$ be a regular cardinal $\geq \aleph_{2}$ and $\lambda$ a regular cardinal $<\kappa$. Then for every ordinal $\theta$ such that $\theta^{+}<\kappa$ the set

$$
\left\{\beta \in \operatorname{Cof}_{\lambda} \cap \kappa \mid \operatorname{ot}\left(C_{\beta}\right) \geq \theta\right\}
$$

is stationary in $\kappa$.

Proof Let $C \subseteq \kappa$ be closed unbounded in $\kappa$. Let $\mu=\max \left(\lambda, \theta^{+}\right)$which is an uncountable regular cardinal $<\kappa$. Take a singular limit point $\gamma$ of $C$ of cofinality $\mu$. Then $C \cap C_{\gamma}$ is closed unbounded in $\gamma$ of ordertype $\geq \mu$. Take $\beta$ to be a singular limit point of $C \cap C_{\gamma}$ such that $\operatorname{cof}(\beta)=\lambda$ and $\operatorname{ot}\left(C \cap C_{\gamma} \cap \beta\right) \geq \theta$. By the coherency property 3 (c), $C_{\beta}=C_{\gamma} \cap \beta$. Thus $\beta \in C \cap\left\{\beta \in \operatorname{Cof}_{\lambda} \cap \kappa \mid\right.$ ot $\left.\left(C_{\beta}\right) \geq \theta\right\} \neq \emptyset$.

Note that $\left(S_{n}\right)_{n<\omega}$ with

$$
S_{n}=\left\{\beta \in \operatorname{Cof}_{\omega_{1}} \cap \aleph_{n+3} \mid \operatorname{ot}\left(C_{\beta}\right) \geq \aleph_{n+1}\right\}
$$

is a sequence of stationary sets to which we could apply the MS-principle.

\section{Hyperfine Singularizations}

Let $\beta$ be a singular ordinal in $L$. We shall assign to $\beta$ a level of the fine structural hierarchy and a parameter which canonically witness the singularity of $\beta$. We use the hyperfine hierarchy of S. D. FrIEDMAN and the first author [5] where the same singularizations were used in the proof of global square.

The hyperfine structural hierarchy refines Gödel's $L_{\alpha}$-hierarchy. The levels of the hierarchy are indexed by locations $s=\left(\alpha, \varphi_{m}, \vec{x}\right)$ where $\alpha \in \operatorname{Ord}$, $\varphi_{m}\left(v_{0}, \ldots, v_{k-1}\right)$ is an $\in$-formula, and $\vec{x}=x_{1}, \ldots, x_{k-1} \in L_{\alpha} .\left(\varphi_{m}\right)_{m<\omega}$ is an appropriate list of all $\in$-formulas. Then

$$
L_{s}=\left(L_{\alpha}, \in,<_{L}, I, N, S, S_{\varphi_{0}}^{L_{\alpha}}, \ldots, S_{\varphi_{m}}^{L_{\alpha}} \uparrow \vec{x}, \emptyset, \emptyset, \ldots\right) ;
$$

Here, $<_{L}$ is the canonical well-ordering of $L, \quad I, N, S$ are an interpretation function, a naming function, and a Skolem function respectively for $L ; S_{\varphi_{i}}^{L_{\alpha}}$ is a Skolem function for $\varphi_{i}$ computed in $L_{\alpha}$. Moreover the last function $S_{\varphi_{m}}^{L_{\alpha}}$ is restricted to arguments $\vec{y}$ which are lexicographically smaller than $\vec{x}$, where the lexicographical order $<_{\text {lex }}$ is derived from $<_{L}$. 
The locations are well-ordered lexicographically by $\tilde{<}$. For each $L_{s}$ there is a hulling operator $L_{s}\{.\} ; L_{s}\{X\}$ is the smallest substructure of $L_{s}$ which contains $X$. The basic fine structural laws of $\left(L_{s}\right)$ and the associated hulling operations are described in [5].

For a given limit ordinal $\beta$ which is singular in $L$ we describe its singularization; in view of the intended applications we also assume that $\operatorname{cof}(\beta) \geq \omega_{1}$. There is a location $s=(\gamma, \varphi, \vec{x})$ and a finite set $p \subseteq L_{\gamma}$ such that $\gamma \geq \beta$ and

(1) $\left\{\bar{\beta}<\beta \mid \bar{\beta}=\beta \cap L_{s}\{\bar{\beta} \cup p\}\right\}$ is bounded below $\beta$.

We say that $\beta$ is semi-singularized at $\left(L_{s}, p\right)$. Let $s=s(\beta)$ be the $\tilde{<}$-minimal location such that $\beta$ is semi-singularized at $\left(L_{s}, p\right)$ for some $p$. Then let $p=p(\beta)$ be a finite set such that $\left(L_{s(\beta)}, p\right)$ semi-singularizes $\beta$ where $p$ is minimal with respect to the $<_{*}$-wellordering of finite subsets of $L: p<_{*} q \leftrightarrow \exists z \in q \backslash p \forall u\left(u<_{L}\right.$ $z \rightarrow(u \in p \leftrightarrow u \in q))$. of $\beta$.

(2) $\left(L_{s(\beta)}, p(\beta)\right)$ exists and semi-singularizes $\beta$; it is called the $L$-singularization

We give some more information about the $L$-singularization. Note that by $\operatorname{cof}(\beta) \geq \omega_{1}$ we are in the "generic case" of [5].

(3) $s(\beta)=(\gamma, \varphi, \vec{x}) \neq\left(\gamma, \varphi_{0}, \overrightarrow{0}\right)$.

(4) There is $\alpha_{0}=\alpha_{0}(\beta)<\beta$ minimal such that $L_{s}\left\{\alpha_{0} \cup p\right\}$ is unbounded below s, i.e., for all $\vec{y}<_{\text {lex }} \vec{x},|\vec{y}|=|\vec{x}|$ there is $\vec{z} \in L_{s}\left\{\alpha_{0} \cup p\right\},|\vec{z}|=|\vec{x}|$ such that $\vec{y}<_{\text {lex }} \vec{z}$; in case that $\vec{x}=\overrightarrow{0}$ we have to require instead that $L_{s}\left\{\alpha_{0} \cup p\right\}$ is cofinal in $\left(L_{s},<_{L}\right)$.

In the construction of the canonical $\square$-sequence $C_{\beta}$ some ordinal $\alpha \leq \alpha_{0}$ will be used as a "steering ordinal". As a brief sketch, we want to define the $C_{\beta}$ sequence with reference to a cofinalising sequence in the location $s$. If $\alpha_{0}$ is a limit ordinal, then we shall take $\alpha_{0}$ itself as $\alpha$. Otherwise $\alpha_{0}=\alpha_{0}^{\prime}+1$, and we have some $\alpha_{1}<\alpha_{0}^{\prime}$ so that $L_{s}\left\{\alpha_{1} \cup\left\{p, \alpha_{0}^{\prime}\right\}\right\}$ is unbounded below $s$; if $\alpha_{1}>0$ but is $\alpha_{1}^{\prime}+1$, we repeat, and see that $L_{s}\left\{\alpha_{2} \cup\left\{p, \alpha_{0}^{\prime} \alpha_{1}^{\prime}\right\}\right\}$ is unbounded below $s$ for some $\alpha_{2}<\alpha_{1}$. After a finite number $k$ of steps we find that $\alpha_{k}$ is zero (in which case we deduce that the cofinality of $\beta=\omega$ ) or a limit. In the latter case, by recursion on $\iota \leq \alpha_{k}$ we define an increasing sequence of hulls in the location whose suprema below $\beta$ will be the elements of what will ultimately contain the $C_{\beta}$ We thus have bounded the order type of $C_{\beta}$ by this "steering ordinal" $\alpha_{k}(\beta) \leq \alpha_{0}$. Hence

(5) $\operatorname{otp}\left(C_{\beta}\right) \leq \alpha_{0}<\beta$.

This restriction on order types will later conflict with the choice of $\left(S_{n}\right)_{n<\omega}$ described in section 2 and conclude a proof by contradiction. 


\section{Lifting up Singularizations}

The following argument is an upward extensions of embeddings construction as known from the proof of Jensen's Covering Theorem:

Theorem 4 Let $\pi:\left(L_{\beta}, \in\right) \rightarrow\left(L_{\beta^{*}}, \in\right)$ be an elementary cofinal map between $\mathrm{ZF}^{-}$-models. Let $\beta$ be singular in $L$ and $\operatorname{cof}(\beta) \geq \omega_{1}$, let $\left(L_{s}, p\right)$ be the $L$ singularization of $\beta$ as described in the previous paragraph. Then there are a uniquely defined structure preserving map $\pi^{*}: L_{s} \rightarrow L_{s^{*}}$ and a parameter $p^{*}$ satisfying:

a) $\pi^{*} \uparrow L_{\beta}=\pi, \pi^{*} " p=p^{*}$;

b) $\left(L_{s^{*}}, p^{*}\right)$ is the L-singularization of $\beta^{*}$.

Proof. The proof of $\square$ in $L$ shows that $L_{s}$ can be represented as $L_{s}=$ $\bigcup_{i<\tau} L_{s_{i}}\left\{\beta_{i} \cup p\right\}$ for strictly increasing sequences $\left(\beta_{i}\right)_{i<\tau}$ and $\left(s_{i}\right)_{i<\tau}$ converging to $\beta$ and $s$ resp., such that each transitive collapse $\sigma_{i}: M_{i} \cong\left(L_{s_{i}}\left\{\beta_{i} \cup p\right\}, p\right)$ is the singularization of $\beta_{i}$.

For $i \leq j<\tau$ let $\sigma_{i j}=\sigma_{j}^{-1} \circ \sigma_{i}: M_{i} \rightarrow M_{j}$. The minimality of $s$ implies that each $M_{i} \in L_{\beta}$ and $\sigma_{i j} \in L_{\beta}$. $\left(M_{i}\right)_{i<\tau},\left(\sigma_{i j}\right)_{i \leq j<\tau}$ is a directed system of $L$-singularizations all of whose components are elements of $L_{\beta}$.

We can now map the directed system pointwise to $L_{\beta^{*}}$ : for $i<\tau$ let $M_{i}^{*}=$ $\pi\left(M_{i}\right)$ and $\sigma_{i j}^{*}=\pi\left(\sigma_{i j}\right) .\left(M_{i}^{*}\right)_{i<\tau},\left(\sigma_{i j}^{*}\right)_{i \leq j<\tau}$ is a commutative system of $L$ singularizations for the ordinals $\beta_{i}^{*}=\pi\left(\beta_{i}\right)$.

(1) The direct limit of $\left(M_{i}^{*}\right)_{i<\tau},\left(\sigma_{i j}^{*}\right)_{i<j<\tau}$ is well founded.

Proof. The indexing ordinal $\tau$ has cofinality $\geq \omega_{1}$. So any descending $\omega$ sequence in the direct limit is already represented in some $M_{j}^{*}$ with $j<\tau$. But $M_{j}^{*}$ is transitive.

Let $M^{*},\left(\sigma_{i}^{*}\right)_{i<\tau}$ be the direct limit of the system $\left(M_{i}^{*}\right),\left(\sigma_{i j}^{*}\right)$. An argument similar to the proof of the condensation theorem in [5] shows that $M^{*}$ is a level of the hyperfine hierarchy, say $M^{*}=L_{s^{*}}$. Define the map $\pi^{*}: L_{s} \rightarrow L_{s^{*}}$ by $\sigma_{i}(z) \mapsto \sigma_{i}^{*}(\pi(z))$. $\pi^{*}$ is a homomorphism by general facts about direct limits. If $z \in L_{\beta}$, then $\sigma_{i}(z)=z$ for sufficiently high $i<\tau$, and so

(2) $\pi^{*} \supseteq \pi$.

Let $p^{*}=\pi^{*}$ " $p$.

(3) $\pi^{*}: L_{s} \rightarrow L_{s^{*}}$ is cofinal with respect to the well-ordering $\tilde{<}$ of locations.

Proof. The location $s^{*}$ is determined as the $\tilde{<}$-minimal location such that $\sigma_{i}^{*}: M_{i}^{*} \rightarrow M^{*}$ is a well-defined homomorphism. This property is equivalent to: for all $i<\tau$ and $M_{i}^{*}=L_{s_{i}^{*}}$ and for all $t \tilde{<} s^{*}$ holds $\sigma_{i}^{*}(t) \tilde{<} s^{*}$.

Consider $r^{*} \tilde{<} s^{*}$. Then take $i<\tau, M_{i}^{*}=L_{s_{i}^{*}}$ and some $t_{0} \tilde{<} s_{i}^{*}$ such that $r^{*} \tilde{\leq} \sigma_{i}^{*}\left(t_{0}\right)$.

Take $j, i<j<\tau$ such that $s_{i} \in L_{s_{j}}\left\{\beta_{j} \cup p\right\}$. Let $s_{i}=\sigma_{j}\left(s_{j}^{\prime}\right), M_{i}=L_{\tilde{s}_{i}}$. Then

$$
\forall t \tilde{<} \tilde{s}_{i}: \sigma_{i j}(t) \tilde{<} s_{i}^{\prime}, \quad \forall t \tilde{<} s_{i}^{*}: \sigma_{i j}^{*}(t) \tilde{<} \pi\left(s_{i}^{\prime}\right) ; \quad \forall t \tilde{<} s_{i}^{*}: \sigma_{i}^{*}(t) \tilde{<} \sigma_{j}^{*}\left(\pi\left(s_{i}^{\prime}\right)\right)=\pi^{*}\left(s_{i}\right) .
$$


In particular: $r^{*} \tilde{\leq} \sigma_{i}^{*}\left(t_{0}\right) \tilde{<} \pi^{*}\left(s_{i}\right)$, as required.

(4) $\left(L_{s^{*}}, p^{*}\right)$ is the $L$-singularization of $\beta^{*}$.

Proof. Take $\delta<\beta$ such that $\left\{\bar{\beta}<\beta \mid \bar{\beta}=\beta \cap L_{s}\{\bar{\beta} \cup p\}\right\} \subseteq \delta$. Set $\delta^{*}=\pi(\delta)$. We claim that $\left\{\eta<\beta^{*} \mid \eta=\beta^{*} \cap L_{s^{*}}\left\{\eta \cup p^{*}\right\}\right\} \subseteq \delta^{*}$. Let $\eta \geq \delta^{*}$. Take $\bar{\beta}<\beta$ minimal such that $\pi(\bar{\beta}) \geq \eta$. Then $\bar{\beta} \geq \delta$ and $\bar{\beta} \nsubseteq \beta \cap L_{s}\{\bar{\beta} \cup p\}$. Take an $L_{s}$-term $t$ and $\vec{x} \subseteq \bar{\beta}$ such that $\bar{\beta} \leq t^{L_{s}}(\vec{x}, p)<\beta$. Since $\pi^{*}$ is a homomorphism,

$$
\eta \leq \pi(\bar{\beta}) \leq t^{L_{s^{*}}}\left(\pi(\vec{x}), p^{*}\right)<\beta^{*} \text {, and } \pi(\vec{x}) \subseteq \pi^{\prime \prime} \bar{\beta} \subseteq \eta .
$$

Hence $\eta \neq \beta^{*} \cap L_{s^{*}}\left\{\eta \cup p^{*}\right\}$, and $s^{*}$ satisfies the semi-singularity property for $\beta^{*}$.

To show that $s^{*}$ is minimal semi-singularizing $\beta^{*}$ consider $r^{*} \tilde{<} s^{*}$. By the cofinality property (3) take $r \tilde{<} s$ such that $r^{*} \tilde{<} \pi^{*}(r)$. By the minimality of $s$, $\left\{\bar{\beta}<\beta \mid \bar{\beta}=\beta \cap L_{r}\{\bar{\beta} \cup p\}\right\}$ is unbounded in $\beta$. Let $\left.\bar{\beta}<\beta, \quad \bar{\beta}=\beta \cap L_{r}\{\bar{\beta} \cup p\}\right\}$. Take $i<\tau$ such that $r \tilde{<} s_{i}, \quad \bar{\beta}<\beta, \quad r \in L_{s_{i}}\left\{\beta_{i} \cup p\right\}$. Then:

$$
\begin{aligned}
& L_{s_{i}} \models \bar{\beta}=\beta \cap L_{r}\{\bar{\beta} \cup p\}, \\
& M_{i} \models \bar{\beta}=\beta_{i} \cap L_{\sigma_{i}^{-1}(r)}\left\{\bar{\beta} \cup \sigma_{i}^{-1} " p\right\}, \\
& M_{i}^{*} \models \pi(\bar{\beta})=\pi\left(\beta_{i}\right) \cap L_{\pi\left(\sigma_{j}^{-1}(r)\right)}\left\{\pi(\bar{\beta}) \cup \pi\left(\sigma_{i}^{-1} " p\right)\right\} .
\end{aligned}
$$

Apply $\sigma_{i}^{*}$ :

$\pi(\bar{\beta})=\beta^{*} \cap L_{\pi^{*}(r)}\left\{\pi(\bar{\beta}) \cup p^{*}\right\}$, and

$\pi(\bar{\beta})=\beta^{*} \cap L_{r^{*}}\left\{\pi(\bar{\beta}) \cup p^{*}\right\}$. Since the set of such $\pi(\bar{\beta})$ is cofinal in $\beta^{*}, r^{*}$ does not semi-singularize $\beta^{*}$, as required.

Now we examine the properties of $p^{*}$. By construction:

$$
(*) L_{s^{*}}=L_{s^{*}}\left\{\beta^{*} \cup p^{*}\right\} .
$$

Suppose that some $q^{*}<_{*} p^{*}$ also satisfies $(*)$. Then $p^{*}=t\left(\vec{x}, q^{*}\right)$ for some term $t$ and $\vec{x}<\beta^{*}$.

$L_{s^{*}}=\exists \vec{x}<\beta^{*} \exists q^{*}<_{*} p^{*} \quad p^{*}=t\left(\vec{x}, q^{*}\right)$.

This existential property can be pulled back to $L_{s}$ via the directed systems:

$L_{s}=\exists \vec{x}<\beta \exists q<_{*} p \quad p=t(\vec{x}, q)$,

which contradicts the minimal choice of $p$.

The previous proof shows that $\pi^{*}$ is cofinal in the locations. This affects the "steering ordinal" $\alpha_{0}$ as follows:

Lemma 1 In the situation of the previous theorem, $\alpha_{0}\left(\beta^{*}\right) \leq \pi\left(\alpha_{0}(\beta)\right)$ and $\operatorname{otp}\left(C_{\beta^{*}}\right) \leq \pi\left(\alpha_{0}(\beta)\right)$.

\section{Getting $O^{\sharp}$}

Theorem 5 If $\operatorname{MS}\left(\left(\kappa_{n}\right)_{n<\omega}, \omega_{1}\right)$ holds then $O^{\sharp}$ exists.

Proof. Assume $\neg O^{\sharp}$. Without loss of generality we may assume that $\kappa_{0} \geq \aleph_{3}$. Set $\kappa_{\omega}=\sup _{n<\omega} \kappa_{n}$. Define a sequence $\left(S_{n}\right)_{n<\omega}$ of stationary sets as in section 
2: $S_{0}=\operatorname{Cof}_{\omega_{1}} \cap \kappa_{0}, S_{1}=\operatorname{Cof}_{\omega_{1}} \cap \kappa_{1}$, and for $n \geq 2$ :

$$
S_{n}=\left\{\beta \in \operatorname{Cof}_{\omega_{1}} \cap \kappa_{n} \mid \operatorname{otp}\left(C_{\beta}\right) \geq \kappa_{n-2}\right\} .
$$

Take a first-order structure $\mathfrak{A}=\left(L_{\kappa_{\omega}^{+}}, \cdots\right)$ with countable language which has a family of Skolem functions $f_{i}$ for $L_{\kappa_{\omega}^{+}}$, constants $\kappa_{0}, \kappa_{1}, \cdots, \kappa_{\omega}$ and functions $g_{i}, n$ :

$$
g_{i, n}(\vec{x})=\sup \left\{f_{i}(\vec{x}, \vec{y}) \mid \vec{y}<\aleph_{2}\right\} \cap \kappa_{n} .
$$

Applying $\operatorname{MS}\left(\left(\kappa_{n}\right)_{n<\omega}, \omega_{1}\right)$ to $\left(S_{n}\right)_{n<\omega}$ and the structure $\mathfrak{A}$ yields some $X \prec L_{\kappa_{\omega}^{+}}$ such that $\left\{\kappa_{n} \mid n \leq \omega\right\} \subseteq X, \quad \forall n<\omega \sup \left(X \cap \kappa_{n}\right) \in S_{n}$, and $\omega_{2} \subseteq X$. Let $\pi:\left(L_{\delta}, \in\right) \cong(X, \in)$, and $\beta_{n}=\pi^{-1}\left(\kappa_{n}\right)$ for $n \leq \omega$. For each $n<\omega: \beta_{n} \geq \aleph_{2}$ and $\operatorname{cf}\left(\beta_{n}\right)=\omega_{1}$. The Jensen Covering Theorem for $L$ implies that every $\beta_{n}$ is a singular ordinal in $L$. For $n<\omega$ let $\left(L_{s_{n}}, p_{n}\right)$ be the singularization of $\beta_{n}$.

(1) If $s_{n}=(\gamma,-,-)$ then $\gamma \geq \beta_{\omega}$, since inside $L_{\beta_{\omega}}, \beta_{n}$ is a regular cardinal.

(2) $s_{n+1} \tilde{\leq} s_{n}$.

Proof. We show that $s_{n}$ singularizes $\beta_{n+1}$ as well as $\beta_{n}$ :

$L_{s_{n}}=L_{s_{n}}\left\{\beta_{n} \cup p_{n}\right\} \supseteq \beta_{\omega} \supseteq \beta_{n+1} \quad, \quad$ and so

$\left\{\bar{\beta}<\beta_{n+1} \mid \bar{\beta}=\beta_{n+1} \cap L_{s_{n}}\left\{\bar{\beta} \cup p_{n}\right\}\right\} \subseteq \beta_{n}$.

Since $\tilde{<}$ is a well-order there is $n_{0}<\omega$ such that $s_{n_{0}}=s_{n_{0}+1}=s_{n_{0}+2}=\ldots$ Set $s=s_{n_{0}}$.

(3) For $n_{0} \leq n<\omega: p_{n+1} \leq_{*} p_{n}$.

Proof. We show that $p_{n}$ satisfies the property in the definition of $p_{n+1}$.

$L_{s}=L_{s}\left\{\beta_{n} \cup p_{n}\right\}$ and so $L_{s}=L_{s}\left\{\beta_{n+1} \cup p_{n}\right\}$.

Since $<_{*}$ is a well-order there is some $n_{1}<\omega, n_{1} \geq n_{0}$ such that $p_{n_{1}}=$ $p_{n_{1}+1}=p_{n_{1}+2}=\ldots$ Set $p=p_{n_{1}}$. Then $\left(L_{s}, p\right)$ is the $L$-singularization of $\beta_{n_{1}}, \beta_{n_{1}+1}, \ldots$ Let $\alpha=\alpha_{0}\left(\beta_{n_{1}}\right)<\beta_{n_{1}}$ as defined in section 3 . As the location $s$ for singularization of the $\beta_{m}$ is the same for $m \geq n_{1}$, the definition of $\alpha_{0}\left(\beta_{m}\right)$ is independent of $m \geq n_{1}$. Thus $\alpha=\alpha_{0}\left(\beta_{m}\right)$ for $n_{1} \leq m<\omega$. For $\beta=\beta_{n_{1}+2}, \beta^{*}=\sup \left(X \cap \kappa_{n_{1}+2}\right)$, we have

$$
\pi \uparrow L_{\beta}: L_{\beta} \rightarrow L_{\beta^{*}}
$$

cofinally as required in Theorem 4 . Then Lemma 1 yields

$$
\operatorname{otp}\left(C_{\beta^{*}}\right) \leq \pi\left(\alpha_{0}(\beta)\right)=\pi(\alpha)<\pi\left(\beta_{n_{1}}\right)=\kappa_{n_{1}} .
$$

But $\beta^{*} \in S_{n_{1}+2}$ and otp $\left(C_{\beta^{*}}\right) \geq \kappa_{n_{1}}$. Contradiction!

\section{Singularizations in Core Models}

For stronger results, we have to apply core models instead of the inner model $L$. We use models of the form $K=L[E]$ where $E$ is a sequence of measures on ordinals. For Theorem 1 we use the DoDD-JENSEN core model below one 
measurable cardinal [2], (and for Theorem 2 we should have to use core model for sequences of measures [7], where $E$ is a sequence of total and partial measures on $K$, together with the more usual fine structure - rather than hyperfine structure). Since our proofs are dependent on a range of results and techniques from core model theory the further presentation has to omit many details and tries to convey basic ideas. We are forced to make several simplifying assumptions and have to argue by analogy with the $L$-case. The general reference to core model theory is the book [9] by MARTIN ZEMAN.

We use the fine structure as developed by JENSEN, For small core models, where the only measures that appear are of MiTCHELL order 0, an extender $E$ with critical point $\kappa$ is a filter indexed by some $\nu$ which will be the successor cardinal of $\kappa$ in the ultrapower of the model by $E$. For higher core models containing sequences of measures, or extenders proper, then larger indices are used (see [9], Chapter 8 for details).

Subsequently, the letter $K$ stands for the DoDD-JENSEN core model. Global Square is proved in $K$ by carefully assigning singularizing sequences to singular ordinals in $K$. We describe the singularization of an ordinal $\beta$ in terms of the JENSEN fine structure for measure sequences ("mice"). It will consist of a level of the fine structural hierarchy and a parameter(-sequence) which canonically witness the singularity of $\beta$.

Definition 4 Let $M=J_{\alpha}[E]$ be a mouse and let $p \in M$ be some finite parameter. Then $(M, p)$ semi-singularizes $\beta$, if $\{\bar{\beta}<\beta \mid \bar{\beta}=\beta \cap M\{\bar{\beta} \cup p\}\}$ is bounded below $\beta$. Here $M\{X\}$ denotes the fine structural hull of $X$ in $M$. For simplicity, we shall say "singularize" instead of "semi-singularize". $M$ as above is called a canonical singularization of $\beta$ if

a) $M \models " \beta$ is regular" or $\beta=\omega \alpha$;

b) $M=M\left\{\beta \cup p_{M}\right\}$;

c) $\left(M, p_{M}\right)$ singularizes $\beta$ where $p_{M}$ is the standard parameter of $M$.

Again, we only say " $K$-singularization" instead of "canonical singularization".

From a $K$-singularization $M$ of $\beta$ one can readily define a subset $C_{\beta}$ of $\beta$ as in the proof of $\square$ which is cofinal in $\beta$ of ordertype $<\beta$. Let us indicate some elements of that definition. In view of the intended applications we also assume that $\operatorname{cof}(\beta) \geq \omega_{1}$. For simplicity we may assume that the first projectum $\rho_{M}^{1}<\beta$ so that we can use the relatively simple $\Sigma_{1}$-finestructure. There is $\alpha_{0}=\alpha_{0}(\beta)<\beta$ minimal such that $M\left\{\alpha_{0} \cup p_{M}\right\}$ is unbounded in $M$. In the construction of the canonical $\square$-sequence $C_{\beta}$ some ordinal $\alpha \leq \alpha_{0}$ will be used as a "steering ordinal" which will imply that $\operatorname{otp}(C) \leq \alpha_{0}<\beta$. This restriction on order types will later conflict with the choice of $\left(S_{n}\right)_{n<\omega}$ described above and conclude a proof by contradiction. The coherency property of $\square$ is due to the coherency between various $K$-singularizations. 
Lemma 2 If $M$ and $N$ are $K$-singularizations of $\beta$ and $M \uparrow \beta=N \uparrow \beta$ then $M=N$. Also $p_{M}$ is the least parameter $p$ such that $\{\bar{\beta}<\beta \mid \bar{\beta}=\beta \cap M\{\bar{\beta} \cup p\}\}$ is bounded below $\beta$.

Proof Coiterate $M$ and $N$ up to $\tilde{M}$ and $\tilde{N}$. As $M \uparrow \beta=N \uparrow \beta$, and we are here dealing with measure filters, no critical point of any measure used in this coiteration is below $\beta$. Thus if $\tilde{M} \in \tilde{N}$ then $\tilde{N}$ contains a code for $M$ and hence $N \models$ " $\beta$ is singular" which contradicts the definition of a $K$-singularization. Hence $\tilde{M}=\tilde{N}$. By the preservation of standard parameters, $p_{M}$ and $p_{M}$ are both mapped to the standard parameter of $\tilde{M}$. Therefore $M$ and $N$ are both the $\beta$-core of $\tilde{M}$ and thus equal. Assume there is some $p<p_{M}$ such that $\{\bar{\beta}<\beta \mid \bar{\beta}=\beta \cap M\{\bar{\beta} \cup p\}\}$ is bounded below $\beta$. Let $(Q, \bar{p}) \cong(M\{\beta \cup p\}, p)$ be the transitivization. Then $(Q, \bar{p})$ singularizes $\beta$. The uniqueness argument above shows that $Q=M$ and $M=M\{\beta \cup \bar{p}\}$ with $\bar{p}<p_{M}$ which contradicts the minimality of $p_{M}$.

\section{$7 \quad$ Lifting up $K$-Singularizations}

We transfer the upward extensions of embeddings technique to the core model situation:

Theorem 6 Let $\pi:\left(J_{\beta}[\bar{E}], \in\right) \rightarrow\left(J_{\beta^{*}}[E], \in\right)$ be an elementary cofinal map between $\mathrm{ZF}^{-}$-models with $\operatorname{cof}(\beta) \geq \omega_{1}$. Let $M=J_{\alpha}[\widetilde{E}]$ be a K-singularization of $\beta$ which end extends $J_{\beta}[\bar{E}]$, i.e., $\alpha>\beta$ and $\widetilde{E} \uparrow \beta=\bar{E} \uparrow \beta$. Then there is a uniquely defined structure preserving map $\pi^{*}: M \rightarrow M^{*}, M^{*}=J_{\alpha^{*}}\left[\widetilde{E}^{*}\right]$ satisfying:

a) $\pi^{*}\left\lceil J_{\beta}[\bar{E}]=\pi, \pi^{*} " p_{M}=p_{M^{*}}\right.$;

b) $M^{*}$ is the unique $K$-singularization of $\beta^{*}$ satisfying $\widetilde{E}^{*} \uparrow \beta=E \uparrow \beta$.

Proof The proof of $\square$ in $K$ shows that $M$ can be represented as $M=$ $\bigcup_{i<\tau} J_{\alpha_{i}}[\widetilde{E}]\left\{\beta_{i} \cup p_{M}\right\}$ for strictly increasing sequences $\left(\beta_{i}\right)_{i<\tau}$ and $\left(\alpha_{i}\right)_{i<\tau}$ converging to $\beta$ and $\alpha$ respectively, such that each transitive collapse $\sigma_{i}: M_{i} \cong$ $J_{\alpha_{i}}[\widetilde{E}]\left\{\beta_{i} \cup p_{M}\right\}$ is the $K$-singularization of $\beta_{i}$. For $i \leq j<\tau$ let $\sigma_{i j}=\sigma_{j}^{-1} \circ \sigma_{i}$ : $M_{i} \rightarrow M_{j}$. Since $\beta$ is a cardinal in $M$ and by acceptability, each $M_{i} \in J_{\beta}[\bar{E}]$ and each $\sigma_{i j} \in J_{\beta}[\bar{E}] .\left(M_{i}\right)_{i<\tau},\left(\sigma_{i j}\right)_{i \leq j<\tau}$ is a directed system of $K$-singularizations all of whose components are elements of $M$.

We can now map the directed system pointwise to $J_{\beta^{*}}[E]$ : for $i<\tau$ let $M_{i}^{*}=\pi\left(M_{i}\right)$ and $\sigma_{i j}^{*}=\pi\left(\sigma_{i j}\right) .\left(M_{i}^{*}\right)_{i<\tau},\left(\sigma_{i j}^{*}\right)_{i \leq j<\tau}$ is a commutative system of singularizations for the ordinals $\beta_{i}^{*}=\pi\left(\beta_{i}\right)$.

(1) The direct limit of $\left(M_{i}^{*}\right)_{i<\tau},\left(\sigma_{i j}^{*}\right)_{i \leq j<\tau}$ is well founded.

Proof. The indexing ordinal $\tau$ has cofinality $\geq \omega_{1}$. So any descending $\omega$-sequence in the direct limit is already represented in some $M_{j}^{*}$ with $j<\tau$. But $M_{j}^{*}$ is transitive. 
Let $M^{*},\left(\sigma_{i}^{*}\right)_{i<\tau}$ be the direct limit of the system $\left(M_{i}^{*}\right),\left(\sigma_{i j}^{*}\right) . M^{*}$ is a level of a $J$-hierarchy, say $M^{*}=J_{\alpha^{*}}\left[\widetilde{E}^{*}\right]$.

(2) $M^{*}$ is a mouse.

Proof. This runs similar to the proof of (1): if $M^{*}$ were not iterable fine structurally then this would be testified in some $M_{j}^{*}$ with $j<\tau$. But $M_{j}^{*}$ is iterable since $M_{j}$ is iterable and $\pi$ is elementary.

Define the map $\pi^{*}: M \rightarrow M^{*}$ by $\sigma_{i}(z) \mapsto \sigma_{i}^{*}(\pi(z))$. $\pi^{*}$ is a homomorphism by general facts about direct limits. If $z \in J_{\beta}$, then $\sigma_{i}(z)=z$ for sufficiently high $i<\tau$, and so

(3) $\pi^{*} \supseteq \pi$.

(4) $\pi^{*}: M \rightarrow M^{*}$ is $\in$-cofinal.

Let $p^{*}=\pi^{*}$ " $p_{M}$. By the direct limit construction:

(5) $M^{*}=M^{*}\left\{\beta^{*} \cup p^{*}\right\}$.

(6) $p^{*}=p_{M^{*}}$.

Proof. $p^{*} \geq p_{M^{*}}$ If $p^{*}>p_{M^{*}}$ then this would be reflected in some $M_{j}^{*}$ with $j<\tau$ but then the elementarity of $\pi$ would yield the contrary.

(7) $\left(M^{*}, p_{M^{*}}\right)$ singularizes $\beta^{*}$.

Proof. Take $\delta<\beta$ such that $\left\{\bar{\beta}<\beta \mid \bar{\beta}=\beta \cap M\left\{\bar{\beta} \cup p_{M}\right\}\right\} \subseteq \delta$. Set $\delta^{*}=\pi(\delta)$. We claim that $\left\{\eta<\beta^{*} \mid \eta=\beta^{*} \cap M^{*}\left\{\eta \cup p_{M^{*}}\right\}\right\} \subseteq \delta^{*}$. Let $\eta \geq \delta^{*}$. Take $\bar{\beta}<\beta$ minimal such that $\pi(\bar{\beta}) \geq \eta$ : then $\bar{\beta} \geq \delta$ and $\bar{\beta} \not \subset \beta \cap M\left\{\bar{\beta} \cup p_{M}\right\}$. Take a term $t$ and $\vec{x} \subseteq \bar{\beta}$ such that $\bar{\beta} \leq t^{M}\left(\vec{x}, p_{M}\right)<\beta$. Since $\pi^{*}$ is a homomorphism,

$$
\eta \leq \pi(\bar{\beta}) \leq t^{M^{*}}\left(\pi(\vec{x}), p_{M^{*}}\right)<\beta^{*} \text {, and } \pi(\vec{x}) \subseteq \pi^{\prime \prime} \bar{\beta} \subseteq \eta .
$$

Hence $\eta \neq \beta^{*} \cap M^{*}\left\{\eta \cup p^{*}\right\}$, and $M^{*}$ satisfies the semi-singularity property for $\beta^{*}$.

The uniqueness of the $K$-singularization $M^{*}$ follows from Lemma 2 .

We saw in the previous proof that $\pi^{*}: M \rightarrow M^{*}$ is cofinal. This again affects the "steering ordinal" $\alpha_{0}$ as follows.

Lemma 3 In the situation of the previous theorem, $\alpha_{0}\left(\beta^{*}\right) \leq \pi\left(\alpha_{0}(\beta)\right)$ and thus ot $\left(C_{\beta^{*}}\right) \leq \pi\left(\alpha_{0}(\beta)\right)$.

\section{Getting an Inner Model with a Measurable Cardinal}

We modify the proof of Theorem 5 to yield the existence of an inner model with a measurable cardinal. We assume $\operatorname{MS}\left(\left(\kappa_{n}\right)_{n<\omega}, \omega_{1}\right)$ and work with the DoDD-JENSEN core model $K$ under the assumption that there is no inner model with a measurable cardinal. By the Dodd-Jensen covering theorem for $K$ every ordinal $\beta \geq \omega_{2}$ with $\operatorname{cof}(\beta) \leq \omega_{1}$ is singular in $K$. In particular $\kappa_{\omega}=\sup _{n<\omega} \kappa_{n}$ is singular in $K$. Take the sequence $\left(S_{n}\right)_{n<\omega}$ of stationary sets $S_{n} \subseteq \kappa_{n}$ as in the proof of Theorem 5. Define the first-order structure $\mathfrak{A}=\left(H_{\kappa_{\omega}^{+}}^{K}, \cdots\right)$ in analogy to that proof. The mutual stationarity property yields some $X \prec H_{\kappa_{\omega}^{+}}^{K}$ 
such that

$\left\{\kappa_{n} \mid n \leq \omega\right\} \subseteq X, \quad \forall n<\omega \quad\left(\sup X \cap \kappa_{n}\right) \in S_{n}$, and $\omega_{2} \subseteq X$.

Let $\pi:(\bar{K}, \in) \cong(X, \in)$ where $\bar{K}$ is transitive, and $\beta_{n}=\pi^{-1}\left(\kappa_{n}\right)$ for $n \leq \omega$. $\bar{K}$ is a mouse without a total measure. For $n<\omega$ take $M_{n}=\left(J_{s_{n}}[E], p_{n}\right)$ to be the $K$-singularization of $\beta_{n}$ (by which we mean the least location $s_{n}$ in the $K$-hierarchy where we take $\left.E=E^{K}\right)$.

Coiterate the mice $\bar{K}$ and $M_{n}$. $\bar{K}$ comes out below $M_{n}$ because $M_{n}$ has information for singularizing $\beta_{n}$ whereas $\beta_{n}$ is regular in $\bar{K}$. So in the coiteration there is no truncation on the $\bar{K}$-side and $M_{n}$ either is an end-extension of $\bar{K}$, or will coiterate up to one.

(1) If $M_{n}$ is not an end-extension of $\bar{K}$, let $\left(\lambda_{i} \mid i \leq \theta\right)$ be the sequence of critical points of the $M_{n}$-side of the coiteration. Then $\lambda_{\theta} \geq \beta_{\omega}$ and $\beta_{\omega} \notin\left\{\lambda_{i} \mid i \leq \theta\right\}$.

Proof. Suppose $M_{n}$ is not an end-extension of $\bar{K}$. If $\lambda_{\theta}<\beta_{\omega}$ then either the $M_{n}$-side had a total measure on $\lambda_{\theta}$ which $\bar{K}$ does not have, or $M_{n}$ were a proper initial segment of $\bar{K}$. Both possibilities lead to a contradiction.

If $\beta_{\omega}=\lambda_{i}$, then the $i$-th iterate $M_{n}^{i}$ of $M_{n}$ would contain $\mathcal{P}\left(\beta_{\omega}\right) \cap \bar{K}$. Since $\beta_{\omega}$ is singular in $\bar{K}, M_{n}^{i}$ would contain a cofinal subset of $\beta_{\omega}$ of small ordertype. But $\lambda_{i}$ is regular in $M_{n}^{i}$.

So the coiterate $M_{n}^{\theta}$ is the minimal iterate of $M_{n}$ whose critical point is $>\beta_{\omega}$, or is $M_{n}$ itself. In the former case, by (1) there is some maximal $i<\theta$ such that $\lambda_{i}<\beta_{\omega}$. Then the iterate $M_{n}^{i+1}$ is generated from $\lambda_{i}+1$ together with some finite parameter, and the critical point of $M_{n}^{i+1}$ is $>\beta_{\omega}$. So in this former case, $M_{n}^{i+1}$ semi-singularizes all $\beta_{m}$ such that $\lambda_{i+1}<\beta_{m}<\beta_{n}$. However in the latter case, since $M_{n}=M_{n}\left\{\beta_{n} \cup p_{M_{n}}\right\}$ and $O n^{M_{n}} \geq \beta_{\omega}$ it is clear that $M_{n}$ itself semi-singularizes all $\beta_{m}$ for $m \geq n$. This implies:

(2) For all $n<\omega$ there exists $n^{\prime}<\omega, n^{\prime} \geq n$ such that for all $m, n^{\prime} \leq m<\omega$ : $M_{m} \leq{ }^{*} M_{n}$

Since $\leq^{*}$ is a pre-wellorder of mice, one can choose a $\leq^{*}$-minimal element of $\left\{M_{n} \mid n<\omega\right\}$. Choose $n_{0}<\omega$ such that

(3) for all $m, n_{0} \leq m<\omega: M_{m} \leq^{*} M_{n_{0}}$ and $M_{n_{0}} \leq^{*} M_{m}$.

By the properties of the $\leq^{*}$-relation:

(4) $M_{m+1}$ is an iterate of $M_{m}$, for $m \geq n_{0}$.

Then $\left(M_{m}\right)_{m>n_{0}}$ is a subsequence of the $M_{n_{0}}$-side of the coiteration of $\bar{K}$ and $M_{n_{0}}$.

By (1), $\beta_{\omega}$ is not a critical point in that iteration of $M_{n_{0}}$. So there must be some $n_{1}<\omega, \quad n_{1} \geq n_{0}$, so that

(5) $M_{m+1}=M_{m}$, for $m \geq n_{1}$ 
Set $M=M_{n_{1}}$. As in the $L$-case:

(6) $p_{m+1} \leq_{*} p_{m}$, for $m \geq n_{1}$.

By the wellfoundedness of $\leq_{*}$ take $n_{2}<\omega, \quad n_{2} \geq n_{1}$ such that $p=p_{n_{2}}=$ $p_{n_{2}+1}=\ldots$. So $(M, p)$ is a common $K$-singularization of $\beta_{n_{2}}, \beta_{n_{2}+1}, \ldots$ We can then conclude the proof by contradiction as in the proof of Theorem 5 .

\section{References}

[1] K. J. Devlin and R. B. Jensen. Marginalia to a theorem of Silver. In A. Oberschelp G. H. Müller and K. Potthoff, editors, $\models I S I L C$ Logic Conference, 1974, number 499 in Lecture Notes in Mathematics, pages 115-142. Springer, 1975.

[2] A.J. Dodd. The Core Model, volume 61 of London Mathematuical Society Lecture Notes in Mathematics. Cambridge University Press, Cambridge, 1982.

[3] J. Cummings M. Foreman and M. Magidor. Canonical structure for the universe of set theory; part 2.

[4] M. Foreman and M. Magidor. Mutually stationary sequences of sets and the non-saturation of the non-stationary ideal on $\mathcal{P}_{\kappa}(\lambda)$. Acta Math.

[5] S. D. Friedman and P. Koepke. An elementary approach to the fine structure of L. Bull. Symb. Logic, 3, no. 4:453-468, 1997.

[6] R. B. Jensen. The fine structure of the constructible hierarchy. AML, 4:229308, 1972.

[7] W. J. Mitchell. The Core Model for Sequences of Measures. I. Math. Proc. Cambridge Philos. Soc., 95, no. 2:229-260, 1984.

[8] P. D. Welch. Combinatorial Principles in the Core Model. D.Phil. thesis, Oxford University, 1979.

[9] M. Zeman. Inner Models and Large Cardinals, volume 5 of Series in Logic and its Applications. de Gruyter, Berlin, New York, 2002. 